This article has been accepted for publication in Monthly Notices of the Royal Astronomical Society (C: 2019 The Authors Published by Oxford University Press on behalf of the Royal Astronomical Society. All rights reserved. 


\title{
Testing the blast-wave AGN feedback scenario in MCG-03-58-007
}

\author{
M. Sirressi, ${ }^{1,2 \star}$ C. Cicone ${ }^{\oplus},{ }^{2}$ P. Severgnini, ${ }^{2}$ V. Braito ${ }^{\oplus,},{ }^{3,4}$ M. Dotti, ${ }^{1}$ R. Della Ceca, ${ }^{2}$ \\ J. N. Reeves, ${ }^{4}$ G. A. Matzeu ${ }^{\oplus}, 3,5$ C. Vignali ${ }^{\oplus 6,7}$ and L. Ballo ${ }^{\oplus 5}$ \\ ${ }^{1}$ Università degli Studi di Milano-Bicocca, Dip. di Fisica G. Occhialini, Piazza della Scienza 3, I-20126 Milano, Italy \\ ${ }^{2}$ INAF - Osservatorio Astronomico di Brera, Via Brera 28, I-20121 Milano, Italy \\ ${ }^{3}$ INAF - Osservatorio Astronomico di Brera, Via Bianchi 46, I-23807 Merate (LC), Italy \\ ${ }^{4}$ Center for Space Science and Technology, University of Maryland Baltimore County, 1000 Hilltop Circle, Baltimore, MD 21250 , USA \\ ${ }^{5}$ European Space Astronomy Centre (ESA/ESAC), Villanueva de la Canada, E-28691 Madrid, Spain \\ ${ }^{6}$ Dipartimento di Fisica e Astronomia, Università di Bologna, via Gobetti 93/2, I-40129 Bologna, Italy \\ ${ }^{7}$ INAF - Osservatorio di Astrofisica e Scienza dello Spazio di Bologna, via Gobetti 93/3, I-40129 Bologna, Italy
}

Accepted 2019 July 30. Received 2019 July 26; in original form 2019 May 31

\begin{abstract}
We report the first Atacama large millimeter/submillimeter array observations of MCG-0358-007, a local $\left(z=0.03236 \pm 0.00002\right.$, this work) AGN $\left(L_{\mathrm{AGN}} \sim 10^{45} \mathrm{erg} \mathrm{s}^{-1}\right)$, hosting a powerful X-ray ultrafast $(v=0.1 c)$ outflow (UFO). The $\mathrm{CO}(1-0)$ line emission is observed across $\sim 18 \mathrm{kpc}$ scales with a resolution of $\sim 1 \mathrm{kpc}$. About 78 per cent of the $\mathrm{CO}(1-0)$ luminosity traces a galaxy-size rotating disc. However, after subtracting the emission due to such rotating disc, we detect with a $\mathrm{S} / \mathrm{N}=20$ a residual emission in the central $\sim 4 \mathrm{kpc}$. Such residuals may trace a low velocity $\left(v_{\mathrm{LOS}}=170 \mathrm{~km} \mathrm{~s}^{-1}\right)$ outflow. We compare the momentum rate $(\dot{P})$ and kinetic power $(\dot{E})$ of such putative molecular outflow with that of the X-ray UFO and find $\dot{P}_{\mathrm{mol}} / \dot{P}_{\mathrm{UFO}} \sim 0.4$ and $\dot{E}_{\mathrm{mol}} / \dot{E}_{\mathrm{UFO}} \sim 4 \times 10^{-3}$. This result is at odds with the energyconserving scenario suggested by the large momentum boosts measured in some other molecular outflows. An alternative interpretation of the residual $\mathrm{CO}$ emission would be a compact rotating structure, distinct from the main disc, which would be a factor of $\sim 10$ 100 more extended and massive than typical circumnuclear discs revealed in Seyferts. In conclusion, in both scenarios, our results rule out the hypothesis of a momentum-boosted molecular outflow in this AGN, despite the presence of a powerful X-ray UFO.
\end{abstract}

Key words: galaxies: active-galaxies: evolution-galaxies: individual (MCG-03-58-007).

\section{INTRODUCTION}

In the context of galaxy formation and evolution studies, it is crucial to understand the physics of the feedback processes from Active Galactic Nucleus (AGN) and Star Formation (SF) activity, which can manifest through powerful galactic outflows (Silk \& Rees 1998; Sijacki et al. 2007). AGN feedback processes may have produced the $\mathrm{M}_{\mathrm{BH}}-\sigma_{*}$ relation (Kormendy \& Ho 2013) through a mechanical interaction between the AGN and the host galaxy. Such an interaction can be provided by powerful and fast winds that develop from the accretion disc and can affect the properties of the interstellar medium (ISM), transporting energy and momentum up to the kpc scales (blast-wave AGN feedback models; e.g. Silk \& Rees 1998; King 2010). Furthermore, feedback mechanisms, from both AGN and SF activity, are often invoked to justify additional observational properties of galaxies: the shape of the baryonic mass function of galaxies (Papastergis et al. 2012), the SF quenching of massive galaxies as indicated by the galaxy colour bimodality (Di Matteo, Springel \& Hernquist 2005; Schawinski et al. 2014), and the $[\alpha / F e]$-enhancement in massive spheroids (Fontanot et al. 2009).

X-ray high signal-to-noise observations led to the discovery of ultrafast $(v>0.1 c)$ outflows (UFO) in 40 percent of the bright nearby local AGN population (Gofford et al. 2015). The X-ray spectra of these sources show one or more deep absorption lines that are best identified with the transitions of highly ionized iron (Reeves, O'Brien \& Ward 2003; Tombesi et al. 2010; Gofford et al. 2015). These transitions, modelled with photoionization models, are blue-shifted with velocities from a few thousand $\mathrm{km} \mathrm{s}^{-1}$ up to tens of thousands of $\mathrm{km} \mathrm{s}^{-1}$ and therefore provide strong evidence for the presence of fast outflowing absorbers at sub-pc scales. Often classified as disc-winds, due to their connection with the accretion disc, X-ray UFOs are a direct manifestation of AGN feedback mechanisms in action.

Another revolution in the study of feedback processes in galaxies has been the recent discovery of massive, galaxy-size, cold molecular outflows with velocities in the range between a few hundreds 
and a few thousands $\mathrm{km} \mathrm{s}^{-1}$ (Feruglio et al. 2010; Fischer et al. 2010). Less massive, multiphase galactic winds were known since the 1980s including ionized, neutral, and molecular gas components (Nakai et al. 1987). However, the massive molecular outflows discovered in the recent decade are estimated to embed up to $\mathrm{M}_{\text {mol }} \sim 10^{10} \mathrm{M}_{\odot}$ of $\mathrm{H}_{2}$ gas (Cicone et al. 2018). Their signature has been found in far-infrared (FIR) spectra featuring prominent $\mathrm{OH}$ and $\mathrm{H}_{2} \mathrm{O}$ P-Cygni profiles (Sturm et al. 2011; Spoon et al. 2013; Veilleux et al. 2013; González-Alfonso et al. 2017) as well as in mm-interferometric observations of molecular emission lines $\left(\mathrm{CO}, \mathrm{HCN}, \mathrm{HCO}^{+}\right)$showing high velocity blue- and red-shifted components clearly deviating from the disc rotation (e.g. Alatalo et al. 2011; Aalto et al. 2012; Cicone et al. 2012; Combes et al. 2013; Cicone et al. 2014; García-Burillo et al. 2014).

Among the theoretical models of AGN-driven outflows that have been proposed, the blast-wave feedback scenario aims to understand the dynamics of both nuclear disc-winds and galacticscale outflows (Silk \& Rees 1998; King 2010; Faucher-Giguère \& Quataert 2012). According to this class of models, a nuclear wind interacts with the ISM, producing a reverse shocked wind and at the same time sweeping up the shocked ambient medium, thus originating a large-scale outflow. One open question regarding this model is whether the outflow is energy-driven (i.e. energyconserving) or momentum-driven. From a theoretical perspective, there is not a well-established agreement on which outflow regime is favoured in nature. If the cooling of the shocked nuclear wind is efficient, the blast-wave models predict a momentum-driven large-scale outflow whose momentum rate is by definition the same as that of the nuclear wind, i.e. $\dot{P}_{\text {out }} \simeq \dot{P}_{\text {wind }} \simeq L_{\mathrm{AGN}} / c$ (King 2010). If the Compton cooling of the shock is instead inefficient, the energy-driven large-scale outflow should show a momentum boost of $\dot{P}_{\text {out }} \gtrsim 10 \cdot \dot{P}_{\text {wind }}$ (Faucher-Giguère \& Quataert 2012). Some of the galaxy-scale molecular outflows detected so far in ultraluminous infrared galaxies (ULIRGs) have a momentum rate that exceeds by an average factor of 20 the momentum flux of the AGN photons (Cicone et al. 2014; Fiore et al. 2017), which would favour a blast-wave energy-driven scenario. For example, in Mrk 231 (Feruglio et al. 2015) and IRAS F11119+3257 (Tombesi et al. 2015), the momentum rate of the molecular outflow appears to exceed that of the X-ray wind by a factor of several. However, such measurements are highly uncertain. Indeed, in IRAS F11119+3257 the molecular outflow energetics revisited using new ALMA data appears more consistent with the momentum conserving scenario (Veilleux et al. 2017). Another notable recent counter example is the quasar PDS 456, where energy-conserving feedback seems to have been ruled out (Bischetti et al. 2019). Increased sample sizes are delivering a much broader range of momentum-boost values (García-Burillo et al. 2015; Pereira-Santaella et al. 2018; Fluetsch et al. 2019).

Another mechanism that can drive galactic outflows, without necessarily requiring the presence of a fast nuclear wind, is based on the AGN radiation pressure acting on the dust that is dynamically coupled at least to the cold phase of the ISM (Costa et al. 2018b). In the multiscattering regime, which requires the dust to be optically thick at IR wavelengths, the momentum rate of the large-scale outflow triggered with such mechanism can exceed the photon momentum (Ishibashi \& Fabian 2015; Bieri et al. 2017; Costa et al. 2018b). Both analytically (Ishibashi, Fabian \& Maiolino 2018) and through hydrodynamical simulations (Costa et al. 2018b), it has been shown that AGN radiative feedback models in luminous quasars $\left(L_{\mathrm{AGN}} \sim 10^{45-47} \mathrm{erg} \mathrm{s}^{-1}\right)$ can explain the observed molecular outflows with a moderate momentum boost.
However, the ability of radiation pressure to significantly accelerate gas-shells is limited to small $(<1 \mathrm{kpc})$ scales where the gas is optically thick in the IR (Costa et al. 2018a). When compared to thermal feedback models, IR radiation pressure can more efficiently eject gas from the bulge and hence regulate SF, but only as long as the gas density is high enough: when a large fraction of gas is blown away, the ISM becomes optically thin and the radiation pressure inefficient (Costa et al. 2018b).

So far, most molecular outflow studies have focused on ULIRGs, whose high infrared luminosity is produced by a combination of AGN and SF activity. In such extreme objects, the intense SF activity, with $S F R \gtrsim 100 \mathrm{M}_{\odot} \mathrm{yr}^{-1}$, is responsible for an important contribution to the feedback processes that is difficult to disentangle from the AGN feedback processes (Cicone et al. 2014). To partially overcome this limitation, in this work we study MCG-03-58-007, which is a local $(z=0.03)$ Seyfert 2, classified as LIRG. The source has a moderate SFR of $20 \mathrm{M}_{\odot} \mathrm{yr}^{-1}$ (Gruppioni et al. 2016), and at the same time hosts a powerful X-ray UFO (Braito et al. 2018), hence we expect AGN feedback processes to dominate over SF feedback. Indeed, the kinetic power of the X-ray wind $\left(\dot{E}_{k}=8\right.$ per cent $\left.\mathrm{L}_{\mathrm{AGN}}\right)$ exceeds the theoretical threshold required to have feedback from an AGN (Hopkins \& Elvis 2010). Such properties make MCG-03-58007 one of the best candidates for isolating the AGN feedback from the SF contribution with the aim of testing the blast-wave feedback models.

The paper is organized as follows. In Section 2.1 we describe the target galaxy and summarize the properties of the X-ray UFO. In Section 2.2 we present the ALMA observations focusing on the $\mathrm{CO}(1-0)$ line. The analysis of the data is presented in Section 3, where we describe the rotating disc model constructed with the software 3D BAROLO. In Section 4 we discuss the interpretation of the results and test the AGN feedback models. In Section 5 we summarize our findings and their implications for the current understanding of the feedback mechanisms at work in galaxies.

Throughout this work, a $H_{0}=70 \mathrm{~km} \mathrm{~s}^{-1} \mathrm{Mpc}^{-1}, \Omega_{\Lambda_{0}}=0.73$, and $\Omega_{m}=0.27$ Cosmology is adopted. At the source redshift $(z=$ $0.03236) 1$ arcsec corresponds to $0.67 \mathrm{kpc}$. Errors are given at $1 \sigma$ confidence level unless otherwise specified, i.e. Section 3.3 and Table 4

\section{OBSERVATIONS}

\subsection{The target galaxy: MCG-03-58-007}

MCG-03-58-007 is a nearby $(z=0.03236$, redshift measurement refined in this work, see Section 3.1) Seyfert type 2 galaxy ( $L_{\mathrm{AGN}} \sim 3 \times 10^{45} \mathrm{erg} \mathrm{s}^{-1}\left({ }^{1}\right)$, for details see Braito et al. 2018) and LIRG $\left(L_{\mathrm{IR}}=2.3 \cdot 10^{11} L_{\odot}\right.$, Gruppioni et al. 2016). A star formation rate of $\mathrm{SFR}=20.1 \pm 0.9 \mathrm{M}_{\odot} \mathrm{yr}^{-1}$ was estimated by Gruppioni et al. (2016), by using the Kennicutt (1998) relation converted to a Chabrier IMF.

Direct evidence for AGN feedback in this source was found with the discovery of an X-ray highly ionized UFO with $v \gtrsim 0.1 c$ and $\log \left(\xi / \mathrm{erg} \mathrm{cm} \mathrm{s}^{-1}\right) \sim 5.5\left({ }^{2}\right)$ in the Suzaku spectrum in 2010 (Braito et al. 2018; Matzeu et al. 2019). Two deep absorption troughs

\footnotetext{
${ }^{1}$ Throughout this work, $L_{\mathrm{AGN}}$ is the bolometric luminosity of the AGN ${ }^{2}$ The ionization parameter is defined as $\xi=\frac{L_{i o n}}{n_{\mathrm{e}} R^{2}}$, where $L_{\text {ion }}$ is the ionizing luminosity in the 1-1000 Rydberg range, $R$ is the distance to the ionizing source, and $n_{\mathrm{e}}$ is the electron density. The units of $\xi \operatorname{are~} \mathrm{erg} \mathrm{cm} \mathrm{s}^{-1} .1 R y=$ $13.6 \mathrm{eV}$.
} 
at $E=7.4 \mathrm{keV}$ and $E=8.5 \mathrm{keV}$, which can be associated with the blue-shifted transitions of Fe XXV and Fe XXVI, strongly indicate the presence of two highly ionized, high column density, and fast outflowing absorbers with velocities of $v_{1} \sim 0.1 c$ and $v_{2}$ $\sim 0.2 c$. Follow-up XMM-Newton and NuSTAR observations in 2015 confirmed the presence of the $v_{1}=0.1 c$ zone of the wind and possibly of an even faster zone (Braito et al. 2018). These observations also showed an occultation event consistent with an increase of the disc-wind opacity.

Braito et al. (2018) compared the energetics of the nuclear wind to the bolometric luminosity of the AGN. They considered only the wind component moving at $v_{1} \sim 0.1 c$, being the only one persistent over time. The mass of the central black hole expected from the $M_{\mathrm{BH}}-\sigma_{*}$ relation is $M_{\mathrm{BH}} \sim 10^{8} \mathrm{M}_{\odot}$. The mass-loss rate of the Xray wind $\left(\dot{M} \sim 1.5 \mathrm{M}_{\odot} \mathrm{yr}^{-1}\right)$ was calculated assuming a biconical geometry for the flow. The kinetic power of the persistent component of the wind is $\dot{E}_{k 1} \sim 2.4 \times 10^{44} \mathrm{erg} \mathrm{s}^{-1}$ ( 8 per cent $L_{\mathrm{AGN}}$ ) and the momentum rate is $\dot{p}_{1} \sim 2 \times 10^{35} \mathrm{~g} \mathrm{~cm} \mathrm{~s}^{-2}$, which is of the same order of $L_{\mathrm{AGN}} / c$. Since the covering factor and the launching radius of the wind are difficult to determine, the uncertainties of the momentum rate and kinetic power are at least $40-50$ per cent.

Besides hosting a stable X-ray UFO, MCG-03-58-007 also hosts an ionized outflow with $v_{\text {ion }} \sim 500 \mathrm{~km} \mathrm{~s}^{-1}$, indicated by the broad blue-shifted wings of the [O III] $4959 \lambda$ and [O III $] 5007 \lambda$ lines visible in the optical spectrum as mentioned by Braito et al. (2018).

\subsection{ALMA observations}

We observed MCG-03-58-007 with ALMA Band 3 during Cycle 4 (Project code: 2016.1.00694.S, PI: P. Severgnini). We used a compact configuration of $4012 \mathrm{~m}$ antennas, in a single pointing with field of view of 75 arcsec (FWHM), which fully covered the target galaxy, as shown in Fig. 1. The observations were carried out on 2017 March 11 with an on-source time of $7.4 \mathrm{~min}$.

We arranged a spectral setup consisting of two spectral windows in each sideband. In the upper sideband we placed one spectral window centred at $v_{\mathrm{obs}}=111.682 \mathrm{GHz}$ for detecting the $\mathrm{CO} \mathrm{J}=1$ 0 (hereafter $\mathrm{CO}(1-0)$ ) line, and another spectral window centred at $v_{\text {obs }}=109.952 \mathrm{GHz}$ to sample the $\mathrm{CN}(\mathrm{N}=1-0, \mathrm{~J}=3 / 2-1 / 2, \mathrm{~J}=1 / 2$ $1 / 2$ ) line doublet, which will be analysed in a separate publication. Both spectral windows in the upper sideband have a width of $1.875 \mathrm{GHz}$ and a resolution of $1.95 \mathrm{MHz}$. In the lower sideband we placed two spectral windows of $2 \mathrm{GHz}$ width and $15.6 \mathrm{MHz}$ resolution centred at $v_{\mathrm{obs}}=97.952 \mathrm{GHz}$ and $v_{\mathrm{obs}}=99.807 \mathrm{GHz}$, to study the $3 \mathrm{~mm}$ continuum emission. Table 1 lists the main parameters of the ALMA CO(1-0) observations. Three calibrators (J2258-2758, Uranus, J2236-1433) were used for fixing the flux density scale, for determining the bandpass response, and for calibrating amplitude and phase of the visibilities of the science target. The calibration pipeline was launched with CASA version 4.7 to produce the visibility files. The data reduction and analysis were performed with CASA version 5.4.

The $\mathrm{CO}(1-0)$ line emission image was deconvolved using the TCLEAN task with Briggs weighting (robust $=0.0$ ). We investigated different masking procedures: in order to create a different mask for every channel, we ultimately used the AUTOMASK parameter. The latter automatically provides a different mask for each channel and minimizes the residual negative sidelobes. The cleaning procedure was carried out over the spectral range corresponding to velocities of $v \in(-1000,+1000) \mathrm{km} \mathrm{s}^{-1}$, on the basis of a preliminary quick imaging that shows no line emission at higher velocities (see also Section 3). We adopted the native spectral resolution of $5 \mathrm{~km} \mathrm{~s}^{-1}$

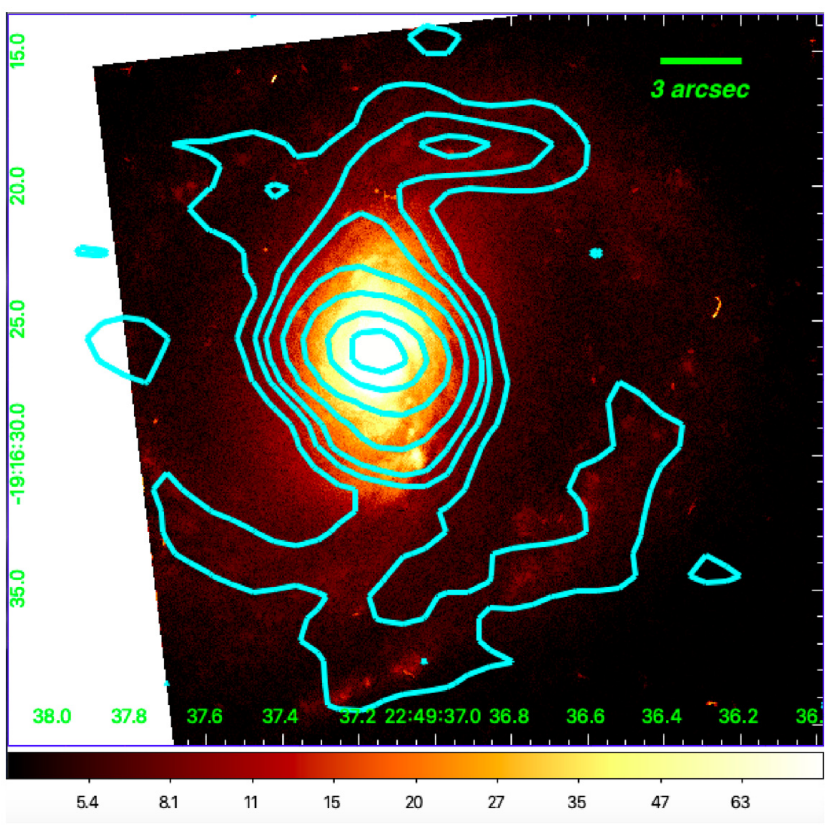

Figure 1. ALMA CO(1-0) emission map (cyan contours) superimposed to the Hubble Space Telescope (HST) optical image (F606W filter) of MCG03-58-007. The horizontal and vertical axes display the Right Ascension (RA) and Declination (Dec), respectively, in sexagesimal units. The $\mathrm{CO}(1-0)$ contours levels correspond to 1-3-5-10-20-40-60 $\sigma$, with $\sigma=0.6 \mathrm{mJy}$. The optical image is scaled with the inverse hyperbolic sine function (asinh). The extension of the galaxy optical emission coincides with the $\mathrm{CO}(1-0)$ line emission and is about 25 arcsec in diameter. The synthesized beam size of the ALMA data is $3.3 \operatorname{arcsec} \times 2.0 \operatorname{arcsec}$.

Table 1. ALMA CO(1-0) observation log.

Target line $\mathrm{CO}(1-0)$ $v_{\text {obs }}$

Date

Array configuration

On-source time

Channel width

Spectral window's width

Rms sensitivity

Synthesized beam
$111.675 \mathrm{GHz}$

2017 March 11

C40-1

$6.70 \mathrm{~min}$

$5.2 \mathrm{~km} \mathrm{~s}^{-1}$ $4992 \mathrm{~km} \mathrm{~s}^{-1}$

$2.2 \mathrm{mJy} /$ beam $/ 5.2 \mathrm{~km} \mathrm{~s}^{-1}$

$3.3 \operatorname{arcsec} \times 2.0 \operatorname{arcsec}$ wide channels. Frequencies were converted to velocities using the optical convention and using the corrected redshift value measured in this work (see Section 3.1). We chose a cleaning threshold equal to the rms per channel $(2.5 \mathrm{mJy})$ and a pixel size of 0.2 arcsec. The primary beam correction was included in the cleaning task to account for the dependence of the sensitivity on the direction within the field of view. The final synthesized beam has an average size of $3.3 \operatorname{arcsec} \times 2.0$ arcsec which corresponds to a physical spatial resolution of $2.2 \mathrm{kpc} \times 1.3 \mathrm{kpc}$. The achieved rms sensitivity is $2.2 \mathrm{mJy} /$ beam $/ 5.2 \mathrm{~km} \mathrm{~s}^{-1}$, as measured from channels free of line emission.

\section{ANALYSIS AND RESULTS}

\subsection{Continuum subtraction and redshift correction}

The $3 \mathrm{~mm}$ continuum emission was subtracted using the task UVCONTSUB from the $u v$ visibilities. For the purpose of continuum 


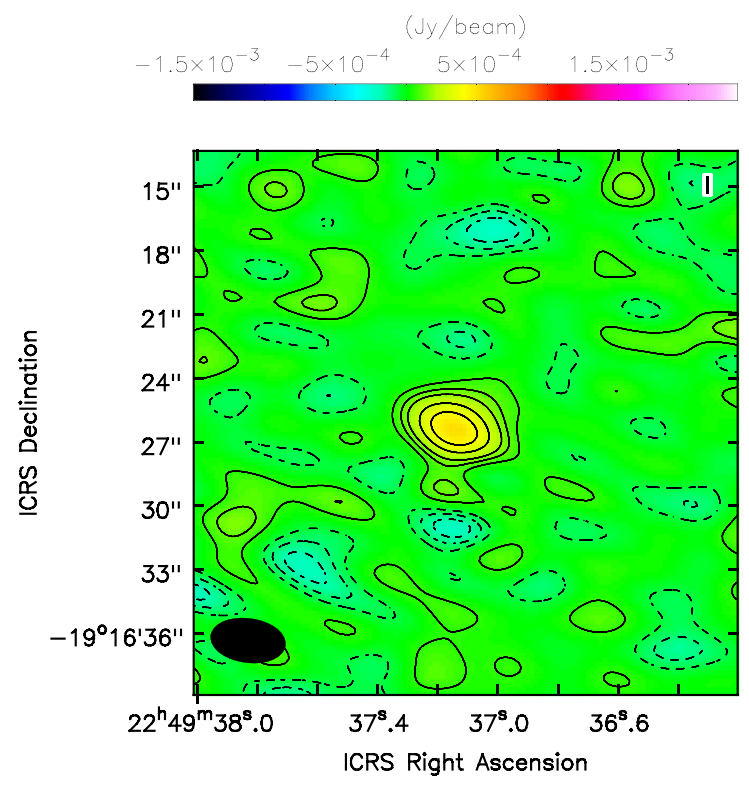

Figure 2. Continuum emission of MCG-03-58-007 at an average $v_{\mathrm{obs}}=$ $99 \mathrm{GHz}$. Two spectral windows, placed on the central frequencies $v_{\mathrm{obs}}=$ $98 \mathrm{GHz}$ and $v_{\mathrm{obs}}=100 \mathrm{GHz}$, free of lines emission, were merged and cleaned for producing this map. The emission is centred on the coordinates of the target, the synthesized beam is represented by the black ellipse in the bottom-left corner of the figure. The black contours levels correspond to $1,2,3,5,7 \sigma_{\mathrm{rms}}$ with $\sigma_{\mathrm{rms}}=0.065 \mathrm{mJy}$. Symmetric negative contours are plotted with dashed lines.

subtraction, we estimated the continuum level from the channels adjacent to the $\mathrm{CO}(1-0)$ line corresponding to velocities $(-2500$, $-500) \mathrm{km} \mathrm{s}^{-1}$ and $(500,2500) \mathrm{km} \mathrm{s}^{-1}$. However, the $3 \mathrm{~mm}$ continuum emission is overall faint compared to the line emission. We measure a continuum flux density of $0.60 \pm 0.10 \mathrm{mJy}$ at $v_{\text {obs }}=99 \mathrm{GHz}$. A map is shown in Fig. 2.

Fig. 3 shows the continuum-subtracted spectrum of the $\mathrm{CO}(1-$ $0)$ line, which is centred at the observed frequency $v_{\text {obs }}=$ $111.658 \mathrm{GHz}$. This frequency is slightly different from that reported in Table $1\left(v_{\mathrm{obs}}=111.675 \mathrm{GHz}\right)$, which was our input $\mathrm{CO}(1-0)$ central frequency inferred from the $\mathrm{CO}(1-0)$ and $\mathrm{CO}(2-1)$ observations carried out with the Swedish European Southern Observatory (ESO) Submillimetre Telescope (SEST) and reported by Strong et al. (2004). These older millimetre data have a spectral resolution of $\Delta v=30 \mathrm{~km} \mathrm{~s}^{-1}$, which is a factor of six larger than that of our ALMA data.

In order to refine the redshift measurement, we used the $\mathrm{CO}(1-0)$ position velocity diagram extracted along an axis with a Position Angle (PA) of $42^{\circ}$ (see Section 3.3), shown in Fig. 4. From this plot we identified the centroid velocity of the rotation curve, which is offset by $v=47 \pm 7 \mathrm{~km} \mathrm{~s}^{-1}$ with respect to the older redshift of $z=$ 0.03220 measured by Strong et al. (2004). By setting the centroid of the $\mathrm{CO}(1-0)$ rotation curve equal to the systemic velocity of the galaxy, we obtain a new redshift estimate of $z=0.03236 \pm 0.00002$ for MCG-03-58-007.

\subsection{Morphology of the CO line emission}

The $\mathrm{CO}(1-0)$ line spectrum extracted from a circular aperture of 25 arcsec shows a complex profile characterized by multiple components (Fig. 3). For this reason we modelled the spectrum with multiple Gaussian functions to measure the total flux and luminosity

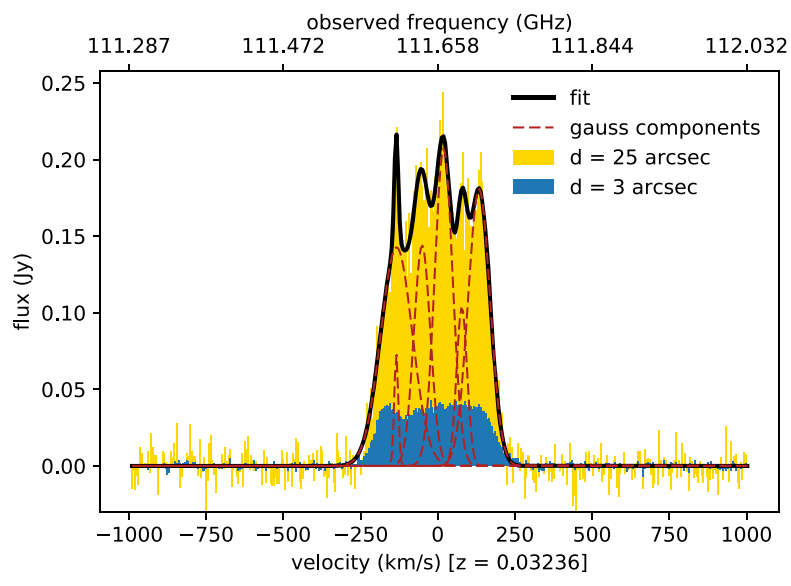

Figure 3. Continuum-subtracted spectrum of the $\mathrm{CO}(1-0)$ line in MCG-0358-007, extracted from circular apertures of $3 \mathrm{arcsec}$ and of $25 \mathrm{arcsec}$ (blue and yellow areas, respectively). The smaller aperture corresponds to the size of synthesized beam, the larger aperture covers the full galaxy extension and is the aperture that maximizes the $\mathrm{CO}$ flux. The black solid line is the best fit of the total spectrum obtained with multiple Gaussian components (red dashed lines). The observed frequency (upper $x$-axis) has been converted to velocity (lower $x$-axis) using the redshift value refined in this work, $z=$ $0.03236 \pm 0.00002$.

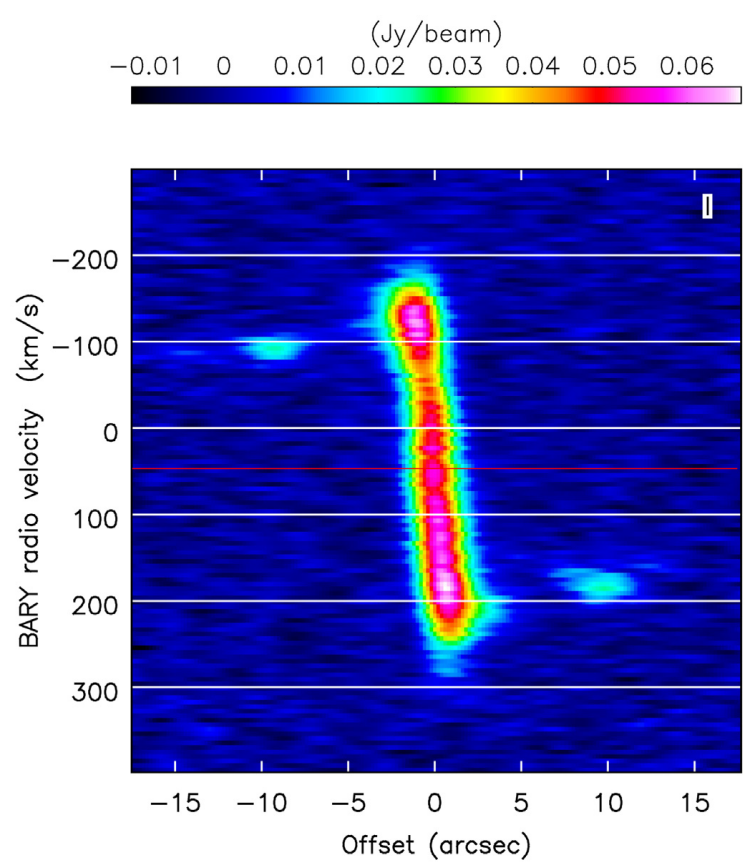

Figure 4. Position-velocity diagram extracted from a beam-wide slit with $\mathrm{PA}=42^{\circ}$. The red horizontal line marks the centroid velocity of the rotation curve, which is offset by $47 \pm 7 \mathrm{~km} \mathrm{~s}^{-1}$ with respect to the input redshift of $z=0.03220$. Our new redshift estimate is therefore $z=0.03236 \pm 0.00002$.

of the $\mathrm{CO}(1-0)$ line. In Table 2 we report the best-fitting parameters for each of the Gaussian components and the values of the $\mathrm{CO}(1-0)$ total flux, luminosity, and total molecular gas mass. We derived a molecular gas mass of $M_{H 2} \simeq(9 \pm 6) \frac{\alpha_{C O}}{3.1} \times 10^{9} \mathrm{M}_{\odot}$ using the formula given by Solomon \& Vanden Bout (2005) with a COto- $\mathrm{H}_{2}$ conversion factor of $\alpha_{C O}=3.1 \pm 2.1 \mathrm{M}_{\odot}\left(\mathrm{K} \mathrm{km} \mathrm{s}^{-1} \mathrm{pc}^{2}\right)^{-1}$. Such conversion factor is the average of the values calculated in nearby star-forming galaxies by Sandstrom et al. (2013) and 
Table 2. Results of a multi-Gaussian fit to the total $\mathrm{CO}(1-0)$ spectrum.

\begin{tabular}{lccc}
\hline $\begin{array}{l}\text { Gaussian } \\
\text { comp. }\end{array}$ & $\begin{array}{c}\mu_{v} \\
\left(\mathrm{~km} \mathrm{~s}^{-1}\right)\end{array}$ & $\begin{array}{c}S_{\text {peak }} \\
(\mathrm{Jy})\end{array}$ & $\begin{array}{c}\sigma_{v} \\
\left(\mathrm{~km} \mathrm{~s}^{-1}\right)\end{array}$ \\
\hline 1 & $-133 \pm 7$ & $0.143 \pm 0.008$ & $73 \pm 6$ \\
2 & $-134 \pm 1$ & $0.073 \pm 0.010$ & $9.2 \pm 1.6$ \\
3 & $-50 \pm 5$ & $0.14 \pm 0.03$ & $38 \pm 8$ \\
4 & $19 \pm 3$ & $0.207 \pm 0.008$ & $41 \pm 8$ \\
5 & $78 \pm 3$ & $0.10 \pm 0.03$ & $24 \pm 4$ \\
6 & $134 \pm 3$ & $0.181 \pm 0.005$ & $50 \pm 3$ \\
\hline Total & $S_{\mathrm{CO}}$ & $L_{\mathrm{CO}}^{\prime}$ & $\begin{array}{l}\mathrm{M}_{\mathrm{H} 2} \\
\text { spectrum }\end{array}$ \\
\hline & $\left.(\mathrm{Jy} \mathrm{km} \mathrm{s})^{-1}\right)$ & $\left(\mathrm{K} \mathrm{km} \mathrm{s}^{-1} \mathrm{pc}^{2}\right)$ & $\left(\mathrm{M}_{\odot}\right)$ \\
\hline & $65 \pm 4$ & $(2.9 \pm 0.5) \times 10^{9}$ & $(9 \pm 6) \frac{\alpha_{C O}}{3.1} \times 10^{9}$ \\
\hline
\end{tabular}

Note. Upper panel: best-fitting parameters of the Gaussian model components showed in Fig. 3: central velocity $\left(\mu_{v}\right)$, flux amplitude $\left(S_{\text {peak }}\right)$, and velocity dispersion $\left(\sigma_{v}\right)$. Lower panel: total $\mathrm{CO}(1-0)$ line flux $\left(S_{\mathrm{CO}}\right)$, total $\mathrm{CO}(1-0)$ line luminosity $\left(L_{\mathrm{CO}}^{\prime}\right)$, and molecular gas mass $\left(M_{H 2}\right)$. Although the amplitude of components 2 and 5 is about half of that of the other components, they are significant at a level $\gtrsim 30 \sigma_{\text {rms }}$. The errors on the Gaussian parameters are given at one standard deviation. The uncertainty on the total flux is dominated by the ALMA calibration error of 6 percent. The error on the luminosity depends also on the error of the Hubble constant: we used $70 \pm 5 \mathrm{~km} \mathrm{~s}^{-1} \mathrm{Mpc}^{-1}$. The molecular gas mass has the largest uncertainty due to the conversion factor which has a precision of 68 percent.

its uncertainty corresponds to the standard deviation of the sample.

In Fig. 5 we show the collapsed map of the $\mathrm{CO}(1-0)$ line emission, obtained by selecting the channels in the range $(-238,213) \mathrm{km} \mathrm{s}^{-1}$, which excludes the $\mathrm{CO}(1-0)$ emission lower than $3 \sigma$. The bulk of the emission is produced in a central circular region of the galaxy of radius $2 \mathrm{kpc}$ and it is detected with a significance of $\sim 50 \sigma$. Some fainter clumps detected at $2-5 \sigma$ are located at larger distances, up to $8 \mathrm{kpc}$ away from the centre.

In Fig. 6 we show the $\mathrm{CO}(1-0)$ channel maps obtained with $\Delta v=50 \mathrm{~km} \mathrm{~s}^{-1}$. As shown in Fig. 6, the red-shifted emission arises from the half portion of the galaxy oriented N-W, while the blueshifted emission comes from the other half oriented S-E. This is the typical pattern of an emitting source that is rotating on a disc, inclined with respect to the line of sight.

At the sensitivity of our data, we did not detect any significant $\mathrm{CO}(1-0)$ emission at velocities $|v|>250 \mathrm{~km} \mathrm{~s}^{-1}$, i.e. higher than the maximum rotation velocity (see also Figs 3 and 4). However, we cannot exclude the presence of a low-velocity outflow whose emission is blended with that of the rotating disc. Therefore in the next section, we model the emission from the rotating gas, subtract it from the ALMA data-cube, and study the morphology and kinematics of any residual $\mathrm{CO}$ emission.

\subsection{Modelling of the main rotating disc}

The $\mathrm{CO}(1-0)$ line emission is dominated by the rotation of the gas in the molecular disc, whose axis is inclined with respect to the line of sight. The typical pattern of such emitting disc (Fraternali et al. 2002) is clearly visible in the PV diagram of Fig. 4 and in the channel maps in Fig. 6. Before modelling the rotating molecular disc, we inferred its geometrical parameters from the intensity (0th moment) and velocity (1st moment) maps of the $\mathrm{CO}(1-0)$ emission, shown in Fig. 7 (left-hand panels). The coordinates of the centre of the disc are determined by performing a Gaussian fit of the central

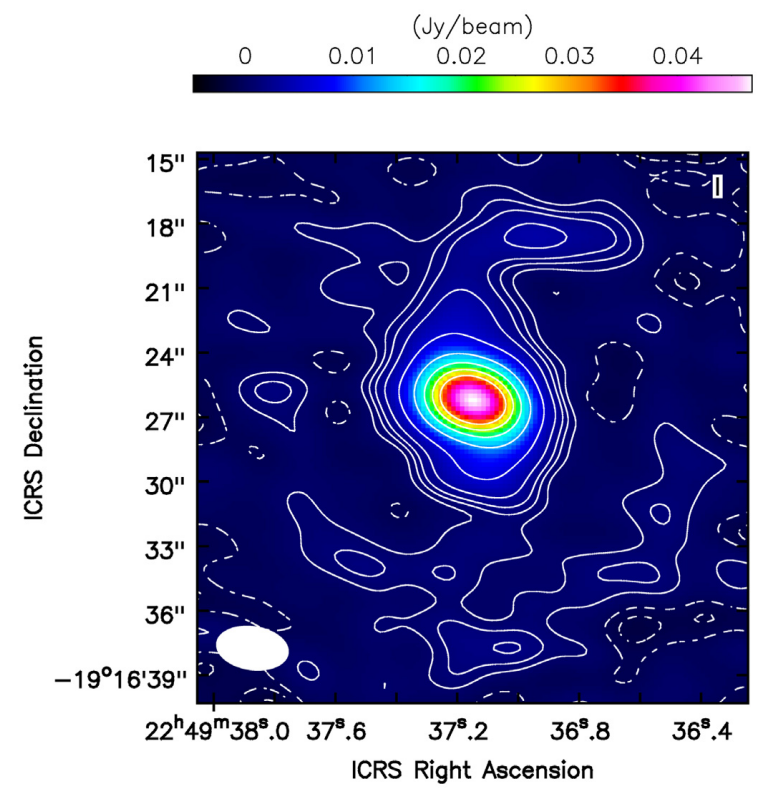

Figure 5. Collapsed map of the $\mathrm{CO}(1-0)$ emission: the flux is summed over the spectral range $(-238,213) \mathrm{km} \mathrm{s}^{-1}$. The white solid lines are the contours level at 1-2-3-5-10-20-30-40-50 $\sigma$, with $\sigma=0.6 \mathrm{mJy}$. Negative contours are also displayed with white dashed lines. The synthesized beam is plotted in the lower left corner in white.

part $(3 \mathrm{kpc})$ of the intensity map. The peak emission is located at $\mathrm{RA}=22^{\mathrm{h}} 49^{\mathrm{m}} 37.16^{\mathrm{s}} \pm 0.07 \mathrm{~s}$, Dec $=-19^{\circ} 16^{\prime} 26.4^{\prime \prime} \pm 1.0^{\prime \prime}$, with errors given by the semiminor axis of the synthesized beam. The inclination angle of the disc has been estimated by measuring the ratio between the minor and major axes of the molecular disc on the velocity map, which features a velocity gradient in the south-west to north-east direction. The inclination angle is defined as the angle between the disc axis and the line of sight (a face-on disc has $i=0^{\circ}$, an edge-on disc has $i=90^{\circ}$ ). We derived a value of $i=37 \pm 10 \mathrm{deg}$, consistent with the broad range of values inferred using the same method for the optical and IR imaging (Jones et al. 2009). The position angle of the major axis of the disc is also derived from the velocity map: we find a value of $P A=42^{\circ}$, measured clockwise starting from the north direction.

We used the software 3D-Based Analysis of Rotating Object via Line Observations (3D BAROLO), developed by Di Teodoro \& Fraternali (2015), for constructing a disc model and fitting its emission to the ALMA data. The main assumption of this model is that all the emitting material of the galaxy is confined to a geometrically thin disc and its kinematics is dominated by pure rotational motion. The typical approach, until recent years, for studying the gas kinematics in a galaxy consisted of fitting the 2D-velocity map with an analytical function for the line of sight velocity. Instead, in the $3 \mathrm{D}$ approach, the fitting technique is based on a Monte Carlo extraction of the positions and velocities of the gas clouds. This permits us to generate the disc one ring at a time and, after the convolution of the disc model with the observational point spread function, to perform the minimization on all the maps (channels) comprising the data-cube.

The input data-cube was obtained by cropping the cleaned, continuum-subtracted ALMA CO(1-0) data-cube ( size $=35$ arcsec $\times 35$ arcsec, velocity range $\left.=(-264,258) \mathrm{km} \mathrm{s}^{-1}\right)$ using the CASA task IMSUBIMAGE. In order to construct the model disc, we used 8 rings of $1.34 \mathrm{kpc}$ width, which is comparable with the angular 

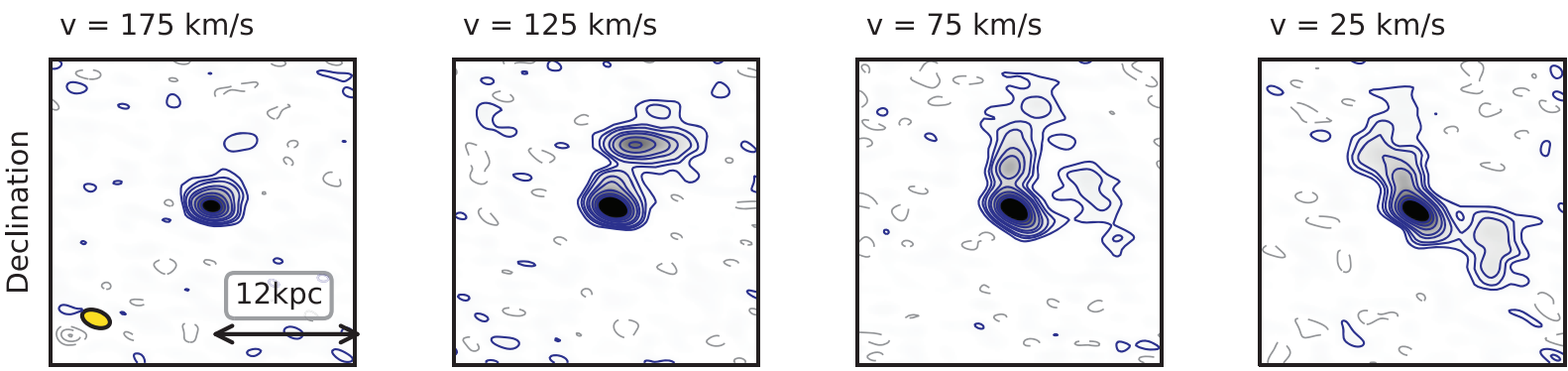

Right Ascension
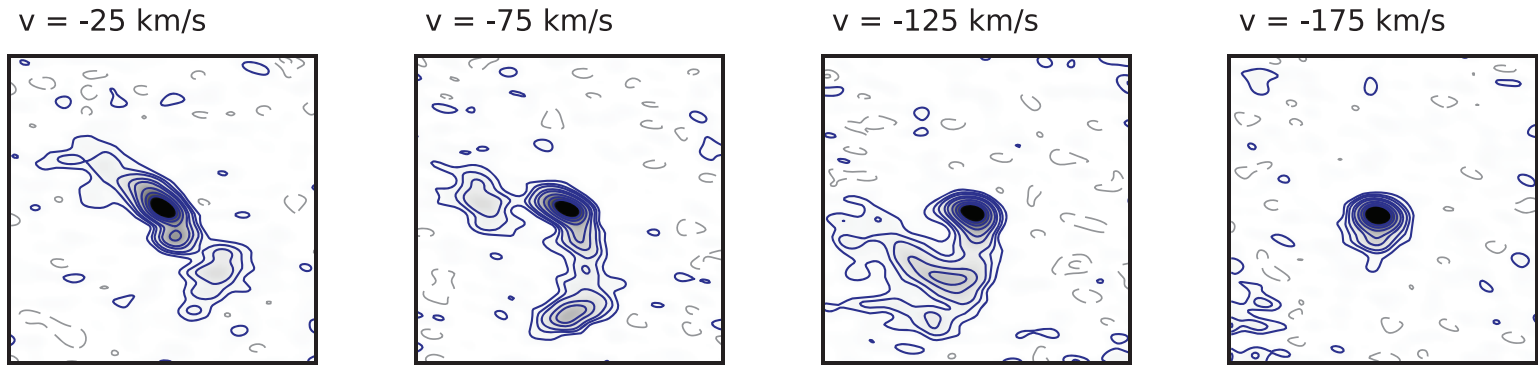

Figure 6. Channel maps of the $\mathrm{CO}(1-0)$ emission, with contours at 3-6-9-15-20-30-40-50 $\sigma_{\mathrm{rms}}, \sigma_{\mathrm{rms}}=0.7 \mathrm{mJy}$ (solid blue lines). Negative contours are plotted with grey dashed lines. Channels have a width of $50 \mathrm{~km} \mathrm{~s}^{-1}$ and their central velocity is displayed in the upper right corner of each image. The synthesized beam is plotted in yellow in the first panel where we also show the physical scale. Each panel is $35 \operatorname{arcsec} \times 35 \operatorname{arcsec}(23.4 \mathrm{kpc} \times 23.4 \mathrm{kpc})$. North and East directions correspond to top and left-hand sides of the panels, as in Fig. 5.
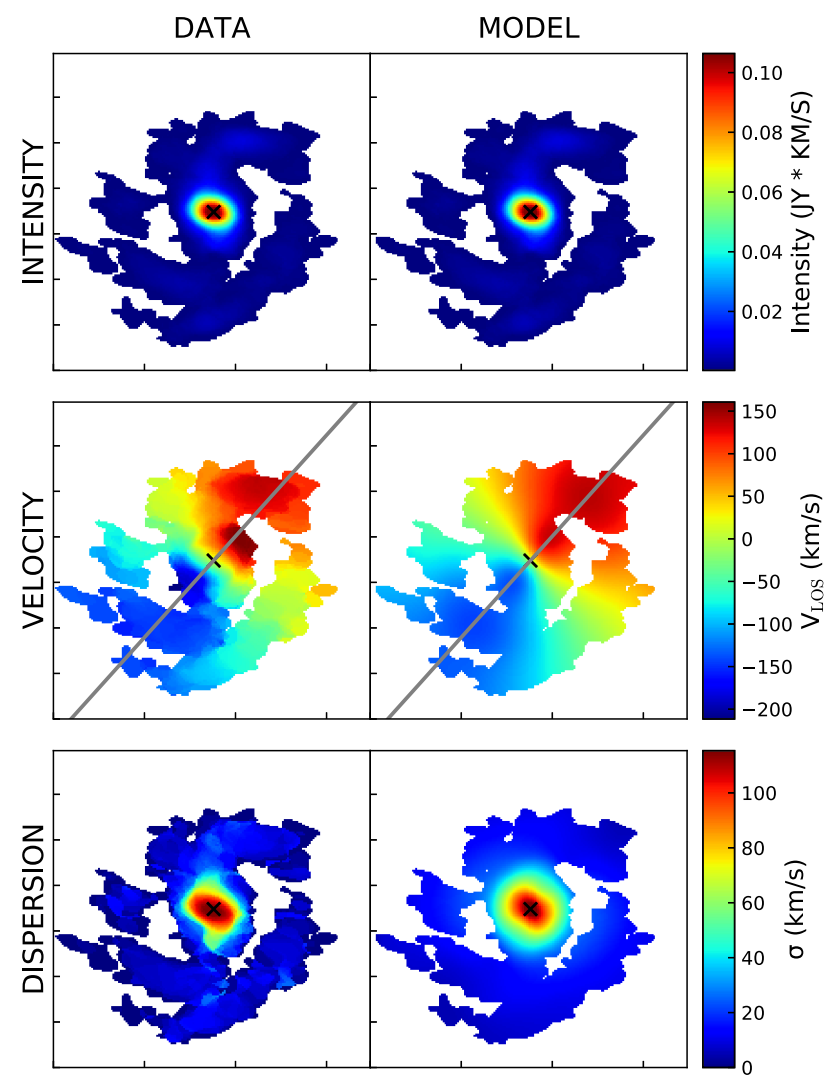

Figure 7. Moment maps of order 0, 1, and 2 (intensity, velocity, and velocity dispersion maps, respectively) of the ALMA CO(1-0) data (lefthand panels) and of the best-fitting model constructed with 3D BAROLO (right-hand panels). A black cross marks the centre of the galaxy. The major axis of the molecular disc is shown by a grey solid line in the velocity map. The white pixels are those with an intensity lower than a threshold of $3 \sigma_{\text {rms }}$. resolution scale of the ALMA data. With such configuration we covered a region of $21.4 \mathrm{kpc}$ in diameter, which includes the fainter distant clumps shown in Fig. 6. The input and output parameters of 3D BAROLO are reported in Table 3.

The fitting was performed considering only pixels with an $\mathrm{S} / \mathrm{N} \gtrsim 3$. In order to estimate the uncertainties on the best-fitting values of the rotation velocities, we employed a Monte Carlo method. After the minimization, the algorithm oversamples the neighbourhood of the minimum in the parameter space, by slightly changing the values of the fitting parameters. The error on each parameter is taken as the variation that produces an increase of 5 per cent in the residuals function. The variation of 5 percent is the default value chosen by the authors of the software BAROLO 3D (Di Teodoro \& Fraternali 2015). In other words, all the possible values of a fitting parameter within its uncertainty guarantee an error of $<5$ per cent on the minimum residual function, and therefore on the best-fit model. ${ }^{3}$

Fig. 7 shows the comparison between the data (left-column panels) and the best-fit model (right-column panels) through the moment (0th, 1st, and 2nd) maps.

In Fig. 8 we show the PV diagrams extracted from the slits along the major and minor axes of the disc (top and bottom panels respectively). In the top panel we superimpose the LOS rotation velocity of each ring of the model disc. Using the best-fitting model, we estimate a total $\mathrm{CO}(1-0)$ flux for the rotating molecular disc of $S_{\mathrm{CO}}^{\text {disk }}=50 \pm 3 \mathrm{Jy} \mathrm{km} \mathrm{s}^{-1}$, from which we derive the CO luminosity of the disc $L_{\mathrm{CO}}^{\prime}=(2.3 \pm 0.4) \times 10^{9} \mathrm{~K} \mathrm{~km} \mathrm{~s}^{-1} \mathrm{pc}^{2}$ and the mass of the molecular disc $M_{\mathrm{H} 2}=(7 \pm 5) \frac{\alpha_{C O}}{3.1} \times 10^{9} M_{\odot}$ (values reported

\footnotetext{
${ }^{3}$ As explicitly stated also in Di Teodoro (2015), there is no direct way in the $3 \mathrm{D}$ approach to calculate the errors of the fitted parameters. Albeit this ' 5 per cent increase' procedure is not optimal and slightly computationally expensive, it returns errors which are in good agreement with those obtained with more standard methods used for the 2D modelling.
} 
Table 3. Parameters of the 3D BAROLO model.

\begin{tabular}{lrrr}
\hline \multicolumn{3}{c}{ Fixed parameters } \\
\hline $\mathrm{i}$ & $\mathrm{PA}$ & $\mathrm{RA}(\mathrm{J} 2000)$ & Dec (J2000) \\
$27^{\circ}$ & $42^{\circ}$ & $12^{\circ} 49^{\mathrm{m}} 37.16^{\mathrm{s}} 26.4^{\prime \prime}$ \\
\hline & & \\
\hline Ring & $\mathrm{R}(\mathrm{kpc})$ & $v_{\text {los }}\left(\mathrm{km} \mathrm{s}^{-1}\right)$ & $v_{\text {rot }}\left(\mathrm{km} \mathrm{s}^{-1}\right)$ \\
1 & 0.67 & $169_{-9}^{+8}$ & $280_{-15}^{+14}$ \\
2 & 2.01 & $164_{-17}^{+17}$ & $273_{-28}^{+28}$ \\
3 & 3.35 & $134_{-12}^{+18}$ & $223_{-20}^{+29}$ \\
4 & 4.69 & $130_{-7}^{+6}$ & $217_{-12}^{+10}$ \\
5 & 6.03 & $94_{-11}^{+10}$ & $156_{-19}^{+17}$ \\
6 & 7.37 & $134_{-6}^{+5}$ & $222_{-10}^{+9}$ \\
7 & 8.71 & $95_{-6}^{+7}$ & $157_{-10}^{+12}$ \\
8 & 10.05 & $127_{-8}^{+7}$ & $211_{-14}^{+12}$ \\
\hline
\end{tabular}

$\mathrm{CO}$ flux and $\mathrm{H}_{2}$ mass of the modelled disc

\begin{tabular}{ccc}
\hline $\begin{array}{c}S_{\mathrm{CO}} \\
\left(\mathrm{Jy} \mathrm{km} \mathrm{s}^{-1}\right)\end{array}$ & $\begin{array}{c}L_{\mathrm{CO}}^{\prime} \\
\left(\mathrm{K} \mathrm{km} \mathrm{s}^{-1} \mathrm{pc}^{2}\right)\end{array}$ & $\begin{array}{c}\mathrm{M}_{\mathrm{H} 2} \\
\left(\mathrm{M}_{\odot}\right)\end{array}$ \\
$50 \pm 3$ & $(2.3 \pm 0.4) \times 10^{9}$ & $(7 \pm 5) \frac{\alpha_{C O}}{3.1} \times 10^{9}$
\end{tabular}

Note. Upper table: values of the input (fixed) parameters of the disc model (inclination angle $i$, position angle of the major axis $P A$, coordinates of the centre of the disc). Middle table: best-fitting rotation parameters for each ring. We report its deprojected radial distance $(R)$, the line of sight velocity $\left(v_{\text {los }}\right)$, and the true rotation velocity $\left(v_{\text {rot }}\right)$. Lower table: total $\mathrm{CO}(1-0)$ flux $\left(\mathrm{S}_{\mathrm{CO}}\right), \mathrm{CO}(1-0)$ luminosity $\left(\mathrm{L}_{\mathrm{CO}}^{\prime}\right)$ and molecular gas mass of the rotating $\operatorname{disc}\left(\mathrm{M}_{\mathrm{H} 2}\right)$.

in Table 3). This corresponds to 78 per cent of the total molecular gas traced by the $\mathrm{CO}(1-0)$ emission detected by ALMA.

\subsection{The $\mathrm{CO}(1-0)$ residual emission}

The output of the 3D BAROLO software is a model-cube of the disc rotation, which we subtracted from the ALMA CO(1-0) line data cube. In Fig. 9 we compare data, model, and residuals using channel maps of $50 \mathrm{~km} \mathrm{~s}^{-1}$ wide channels.

In both the receding and approaching velocities, the residuals show a spatially extended emission detected at the $3 \sigma$ level, which is consistent with low-level residual rotation that is not accounted for by the fit. In addition, in the two symmetric channels at higher velocities $\left(-175 \mathrm{~km} \mathrm{~s}^{-1}\right.$ and $\left.+175 \mathrm{~km} \mathrm{~s}^{-1}\right)$, we detect a compact two-fold structure at much higher significance $(\mathrm{S} / \mathrm{N}=20)$. In Fig. 10 we show a contour map of such compact residual structure, obtained by integrating the residual flux over the spectral ranges $(-222,-148) \mathrm{km} \mathrm{s}^{-1}$ and $(121,195) \mathrm{km} \mathrm{s}^{-1}$.

The blue- and red-shifted portions of such structure can fit inside a rectangle of sides $3.3 \mathrm{kpc} \times 4.7 \mathrm{kpc}$. The centroids of these two regions are $\sim 2.5 \operatorname{arcsec}(1.7 \mathrm{kpc})$ offset from the centre of the galaxy. From these two regions we separately extracted the spectra shown in Fig. 11. We modelled both spectra with a Gaussian and computed the fluxes in Table 4, where we report also the respective luminosities and physical sizes. In Section 4 we discuss two possible interpretations for such residual compact emission that is not accounted for by the modelling of the main large-scale molecular disc.
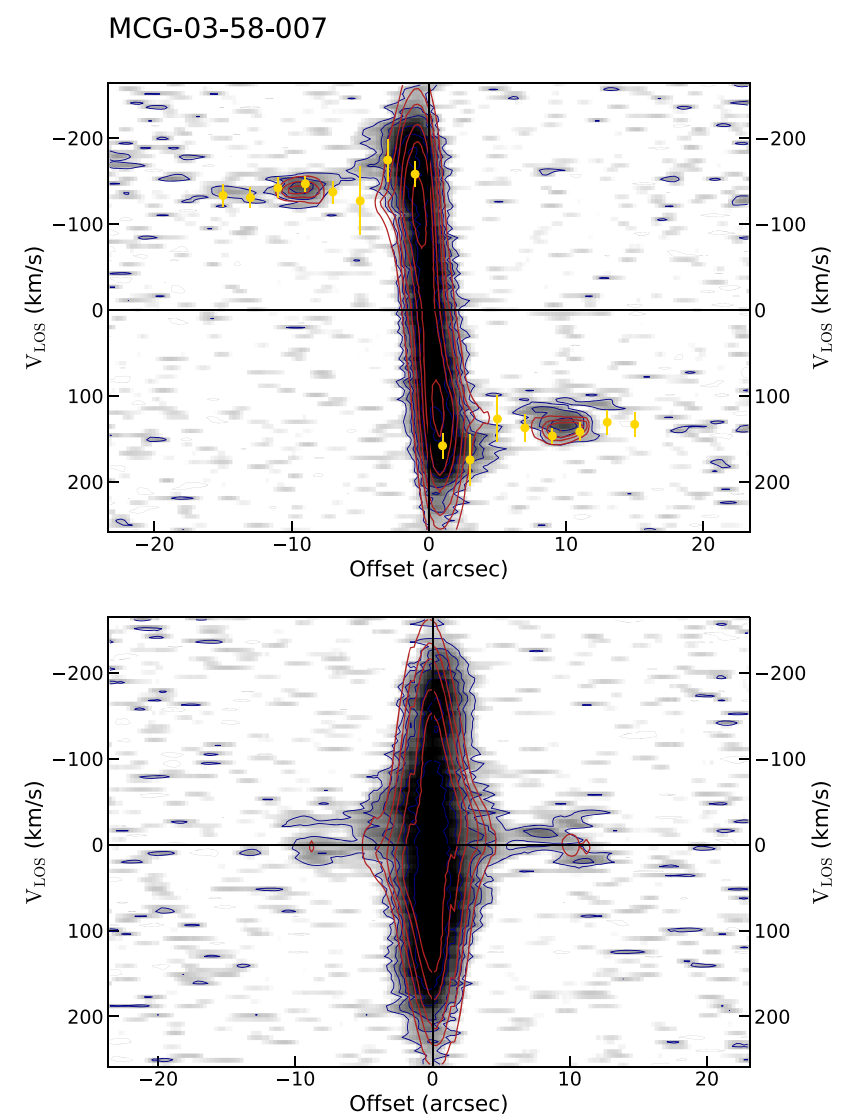

Figure 8. PV diagrams extracted from the data-cube (blue solid contours) and model-cube (red solid contours) using one slit along the major axis (top panel) and another one along the minor axis (bottom panel). The contours level of both the data and the model are at 3-6-9-15-20-30-40-50 $\sigma$. The rotation velocity of each ring of the disc model is plotted with yellow solid dots in the top panel along with the error bars.

\section{DISCUSSION}

Our ALMA CO(1-0) observations of MCG-03-58-007 revealed a rotating galaxy-size molecular disc, whose emission contributes to $\sim 78$ per cent of the total $\mathrm{CO}(1-0)$ flux, in addition to a more compact $(R \lesssim 2 \mathrm{kpc})$ emission that is not consistent with the modelled rotation. This residual emission, which shows a doublelobe blue-shifted southern component and a symmetrical, doublelobe red-shifted northern component (see corresponding map and spectrum shown in Figs 10 and 11), may trace a low-velocity outflow $\left(v \sim 170 \mathrm{~km} \mathrm{~s}^{-1}\right)$. Alternatively, this emission may be due to a rotating structure that is decoupled from the main disc fitted by 3D BAROLO, possibly because it has a different inclination angle or alignment compared to the large-scale rotation. In the next sections we discuss these two possible interpretations.

\subsection{Case I: a low-velocity compact outflow}

We discuss here the hypothesis that the structure in Fig. 10 traces a molecular outflow. This interpretation is supported not only by the detection of a powerful X-ray nuclear UFO in this source (as discussed in Sections 1 and 2.1), but also by the presence of an ionized outflow on scales comparable to the $\mathrm{CO}$ residual emission (Braito et al. 2018). In particular, the 6dF optical spectrum (Jones et al. 2009) of MCG-03-58-007 shows blue-shifted wings in both 

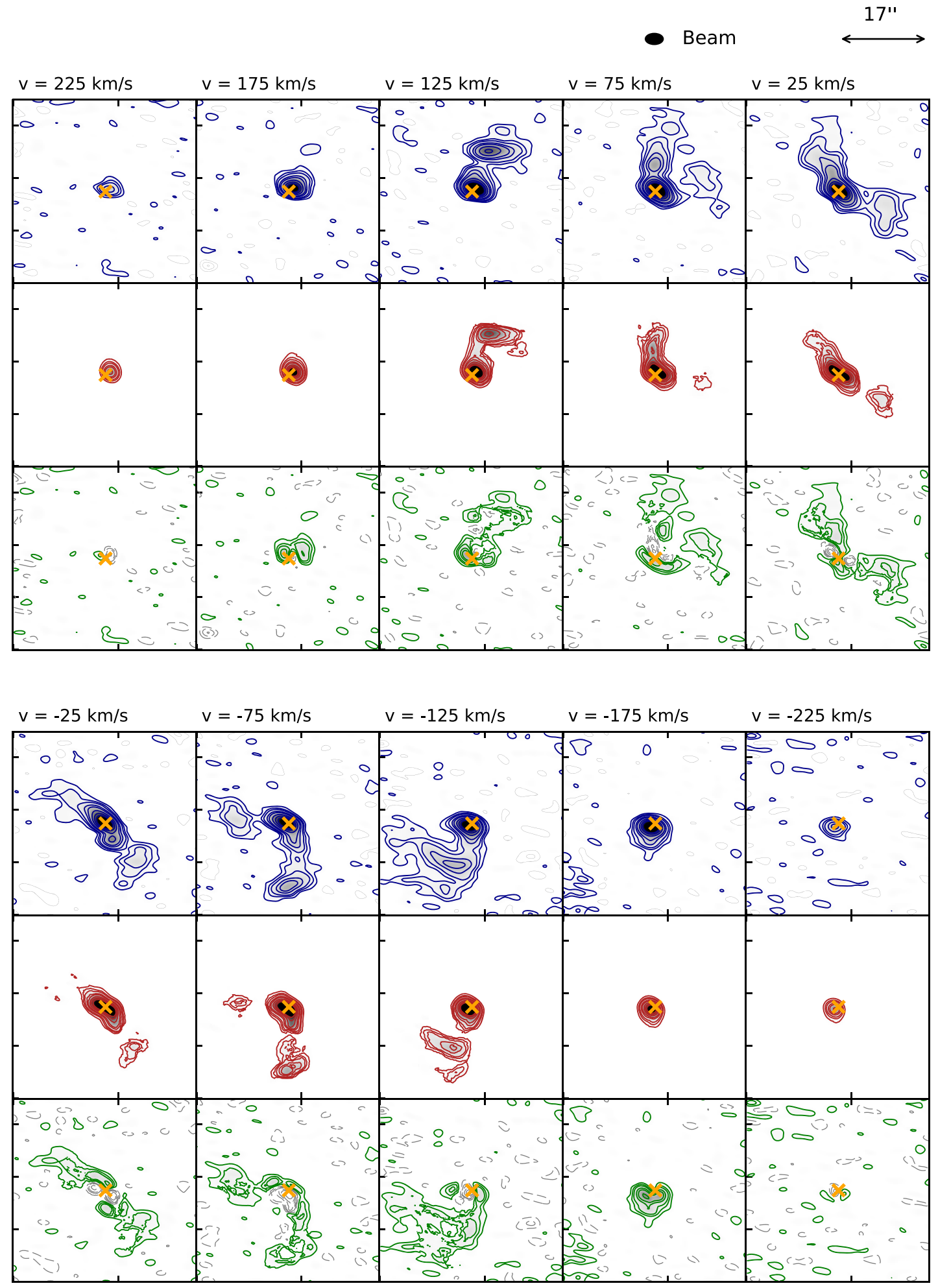

Figure 9. $\mathrm{CO}(1-0) 50 \mathrm{~km} \mathrm{~s}^{-1}$-wide channel maps. From top to bottom rows: data, rotating disc best-fitting model, and residuals (blue, red, and green solid contours, respectively). Contours levels correspond to 3, 6, 9, 15, 20,30,40,50 $\sigma_{\mathrm{rms}}$ with $\sigma_{\mathrm{rms}}=0.7 \mathrm{mJy}$. Symmetric negative contours are also displayed with dashed grey lines. The yellow cross in each panel marks the centre of the disc. Each panel is $35 \operatorname{arcsec} \times 35 \operatorname{arcsec}\left(23.4 \times 23.4 \mathrm{kpc}^{2}\right)$. North and East correspond to the upper and left-hand sides of the panels.

the $[\mathrm{O}$ III $] 4959 \lambda$ and $[\mathrm{O}$ III] $] 5007 \lambda$ emission lines, shifted by about $500 \mathrm{~km} \mathrm{~s}^{-1}$. We note that the $6 \mathrm{dF}$ optical spectrum was extracted using a fibre aperture of $6.7 \operatorname{arcsec}(4.5 \mathrm{kpc})$, which coincides with the region where we detect significant $\mathrm{CO}$ residuals (Fig. 10).
However, we cannot exclude that the ionized wind extends further up to the kpc scales, outside the fibre coverage.

In the hypothesis that the residual emission shown in Fig. 10 traces a low-velocity $\left(v<200 \mathrm{~km} \mathrm{~s}^{-1}\right)$ molecular outflow, we 


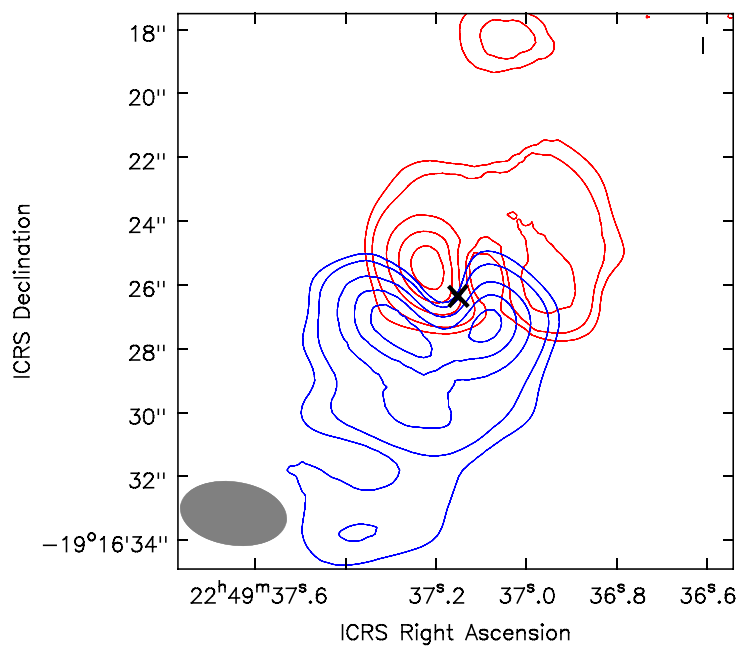

Figure 10. Intensity maps of the blue-shifted (blue solid contours) and red-shifted residual emission (red solid contours) after subtracting the main disc model. The $\mathrm{CO}$ emission was integrated, respectively, over $v \in(-148,-222) \mathrm{km} \mathrm{s}^{-1}$ and $v \in(121,195) \mathrm{km} \mathrm{s}^{-1}$. Contour levels correspond to $3-5-10-15-20 \sigma$. The centre of the galaxy is marked with a black cross. The synthesized beam is plotted in grey at the bottom left corner of the panel.

assume a simple biconical model to account for both the blueshifted and red-shifted emissions that are symmetric with respect to the AGN location (Fig. 10). We derive all the quantities that characterize the outflow separately for the approaching and the receding gas, with the following methods. The outflowing velocities (i.e. $v_{\text {out }}^{\text {blue }}$ and $v_{\text {out }}^{\text {red }}$ ) are estimated from the peaks positions of the bestfitting Gaussians of Fig. 11, reported in Table 4. We estimated the size of the outflow components $(R)$ as the projected extensions of the single residual components (red and blue), also reported in Table 4. The dynamical time of the outflow is calculated as $\tau_{\mathrm{dyn}}=R / v_{\text {out }}$, assuming for simplicity that the axes of the model cones form an angle of $45^{\circ}$ with the line of sight. This is equivalent to assuming that the ratio $R / v_{\text {out }}$ (projected and line-of-sight component for the extension and the velocity, respectively) represents the ratio between the true extension and velocity of the outflow. Realistic deviations of such angle $\left(\delta \theta \lesssim 15^{\circ}\right)$ do not affect the outflow properties beyond their margins of error.

The masses are derived from the residual $\mathrm{CO}(1-0)$ luminosities of Table 4 assuming a CO-to- $\mathrm{H}_{2}$ conversion factor of $\alpha_{\mathrm{CO}}^{\text {out }}=$ $2.1 \mathrm{M}_{\odot}\left(\mathrm{K} \mathrm{km} \mathrm{s}^{-1} \mathrm{pc}^{2}\right)^{-1}$, which is the value calculated by Cicone et al. (2018) for the molecular outflow of NGC $6240 .{ }^{4}$ We cannot quote a formal statistical error for the $\alpha_{\mathrm{CO}}^{\text {out }}$ parameter in MCG-0358-007's putative outflow (and so all the quantities depending on it). However, based on the few observational constraints available for other molecular outflows in galaxies with similar ISM properties and star formation activity as our target (e.g. Leroy et al. 2015; Cicone et al. 2018; Zschaechner et al. 2018), we expect the $\alpha_{\mathrm{CO}}^{\text {out }}$ parameter to be within $\alpha_{\mathrm{CO}}^{\text {out }} \in[0.8,3.4] \mathrm{M}_{\odot}\left(\mathrm{K} \mathrm{km} \mathrm{s}^{-1} \mathrm{pc}^{2}\right)^{-1}$. We note that there is another source, IC5063, for which observations suggest a lower value of $\alpha_{\mathrm{CO}}^{\text {out }}=0.3 \mathrm{M}_{\odot}\left(\mathrm{K} \mathrm{km} \mathrm{s}^{-1} \mathrm{pc}^{2}\right)^{-1}$ (Oosterloo

\footnotetext{
${ }^{4}$ In NGC 6240 the $\alpha_{\mathrm{CO}}^{\text {out }}$ estimated for the outflow is lower than the $\alpha_{\mathrm{CO}}$ estimated for the non-outflowing $\mathrm{H}_{2}$ gas reservoir, as expected if the large $\sigma_{v}$ of the clouds in outflow partially decrease the optical depth of its global low-J CO emission (Cicone et al. 2018)
}

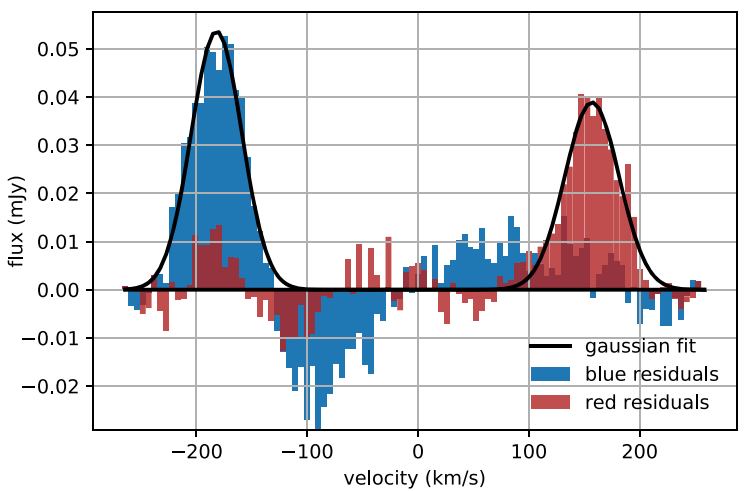

Figure 11. CO spectra extracted from the regions of the blue- and redshifted emission shown in Fig. 10. Both spectra were extracted from a polygon area whose perimeter coincides with the contour line at $5 \sigma$ in Fig. 10. The black solid lines show the two best-fitting Gaussian functions.

et al. 2017). However, IC5063 is a Seyfert 2 hosted by an early-type galaxy, hence its ISM conditions are very different from our target.

The mass-loss rate in this model is given by $\dot{M}_{\text {out }}=M_{\text {out }} / \tau_{\text {dyn }}$. The momentum rate and the kinetic power of the outflow are defined as $\dot{P}_{\text {out }}=\dot{M}_{\text {out }} v_{\text {out }}$ and $\dot{E}_{\text {out }}=\frac{1}{2} \dot{M}_{\text {out }} v_{\text {out }}^{2}$, respectively. Their estimates are corrected for the line-of-sight effects on the velocity, consistently with the assumption of a bi-cone inclination of $45^{\circ}$. All the derived quantities of the total molecular outflow are reported in Table 5, along with the same parameters derived for the nuclear wind detected in the X-ray spectrum (Braito et al. 2018). The velocity, radius, and dynamical time of the outflowing molecular gas are the values averaged between the approaching and receding components.

\subsubsection{Testing the AGN feedback scenario}

The mass-loading factor $\eta \equiv \dot{M}_{\text {out }} / \mathrm{SFR} \sim 3$ indicates that such putative molecular outflow is potentially able to quench the starformation in MCG-03-58-007. The mass-loading factor also suggests that SF alone may not be sufficient at driving such outflow, hence requiring the additional energy from the AGN, as expected from a blast-wave feedback mechanism initiated by the X-ray wind (see also Section 4.1.2). Therefore, MCG-03-58-007 is a good laboratory to test AGN feedback models, which can be done by comparing the energetics of the putative molecular outflow to the energetics of the stable component of the X-ray UFO.

The ratio between the momentum rates of the two outflows is $\dot{P}_{\text {mol }} / \dot{P}_{\mathrm{UFO}} \sim 0.4$. Although this ratio has been obtained following a series of assumptions used to estimate the energetics of the two outflows, it allows us to rule out the presence of a momentumboosted molecular outflow in our target. Furthermore, it is also possible - we note - that a non-negligible fraction of the initial momentum is transferred to the ionized outflow. However, with the current, spatially unresolved, optical data it is not possible to constrain the energetics of the ionized outflow (and, in any case, it is very difficult to constrain the masses and energetics of ionized outflows in absence of an estimate of the electron density, see considerations in e.g. Harrison et al. 2018). Instead the kinetic power of the molecular outflow is three orders of magnitude lower than that of the X-ray wind. Remarkably, this is at tension with a classic energy-conserving blast-wave feedback scenario which predicts not only the kinetic power to be conserved but also the momentum rate of the large scale outflow to be boosted (Faucher- 
Table 4. Properties of the residual CO(1-0) emission shown in Figs 10 and 11 obtained from the spectral fit.

\begin{tabular}{lcccc}
\hline Gaussian & $v\left(\mathrm{~km} \mathrm{~s}^{-1}\right)$ & $S_{\mathrm{CO}}\left(\mathrm{Jy} \mathrm{km} \mathrm{s}^{-1}\right)$ & $L_{\mathrm{CO}}^{\prime}\left(\mathrm{K} \mathrm{km} \mathrm{s}^{-1} \mathrm{pc}^{2}\right)$ & $R(\mathrm{kpc})$ \\
\hline Red & $157 \pm 5$ & $2.39 \pm 0.14$ & $(1.09 \pm 0.17) \times 10^{8}$ & $1.7 \pm 0.7$ \\
Blue & $-182 \pm 5$ & $3.07 \pm 0.18$ & $(1.40 \pm 0.22) \times 10^{8}$ & $1.7 \pm 0.7$ \\
\hline
\end{tabular}

Note. We report the velocity $(v)$, the $\mathrm{CO}$ flux $\left(S_{\mathrm{CO}}\right)$, the CO luminosity $\left(L_{\mathrm{CO}}^{\prime}\right)$, and the projected size $(R)$ for the two components of the residual emission (red and blue). The projected sizes are defined to be the distance of the centroid to the centre of the galaxy and their errors are given by the semiminor axis of the synthesized beam. For the uncertainties of other parameters, see caption of Table 2.

Table 5. Comparison between the molecular outflow and X-ray UFO properties.

\begin{tabular}{|c|c|c|c|c|c|c|c|}
\hline Type & (2) & $\begin{array}{c}R \\
(\mathrm{pc}) \\
(3)\end{array}$ & $\begin{array}{c}\tau_{\text {dyn }} \\
\left(10^{7} \mathrm{yr}\right) \\
(4)\end{array}$ & $\begin{array}{c}M_{\text {out }} \\
\left(10^{8} \mathrm{M}_{\odot}\right) \\
(5)\end{array}$ & $\begin{array}{c}\dot{M}_{\text {out }} \\
\left(\mathrm{M}_{\odot} \mathrm{yr}^{-1}\right) \\
(6)\end{array}$ & $\begin{array}{c}\dot{P}_{\text {out }} \\
\left(\mathrm{g} \mathrm{cm} \mathrm{s}^{-2}\right) \\
(7)\end{array}$ & $\begin{array}{c}\dot{E}_{\text {out }} \\
\left(\mathrm{erg} \mathrm{s}^{-1}\right) \\
(8)\end{array}$ \\
\hline X-ray UFO & $0.075 c$ & $1.7 \times 10^{-3}$ & - & - & 1.5 & $2 \times 10^{35}$ & $2.4 \times 10^{44}$ \\
\hline
\end{tabular}

Notes. (1) Outflow type; (2) outflow velocity (LOS component); (3) projected extension of the outflow; (4) dynamic time of the outflow; (5) outflowing mass; (6) mass-loss rate; (7) momentum rate; (8) kinetic power.

$\left(^{*}\right)$ The $\mathrm{H}_{2}$ outflow energetics depends on the $\alpha_{\mathrm{CO}}^{\text {out }}$ conversion factor, which we assumed equal to $2.1 \mathrm{M}_{\odot}\left(\mathrm{K} \mathrm{km} \mathrm{s}^{-1} \mathrm{pc}^{2}\right)^{-1}$. As explained in Section 4, based on the few observational constraints available for $\alpha_{\mathrm{CO}}^{\text {out }}$ in outflow, we expect this value to be comprised within $\alpha_{\mathrm{CO}}^{\text {out }} \in[0.8,3.4] \mathrm{M}_{\odot}\left(\mathrm{K} \mathrm{km} \mathrm{s}^{-1} \mathrm{pc}^{2}\right)^{-1}$.

Giguère \& Quataert 2012). The efficiency factor, which we define as the ratio between the kinetic power of the molecular outflow to the X-ray wind, is $\dot{E}_{\text {mol }} / \dot{E}_{\mathrm{UFO}} \sim 4 \times 10^{-3}$, lower than the range found by Mizumoto, Izumi \& Kohno (2019) for a sample of six Seyfert galaxies, i.e. from $\sim 7 \times 10^{-3}$ to $\sim 1$. Our results are consistent with the model of a momentum-conserving outflow where the nuclear wind is able to dissipate the energy via a radiative shock (King 2010). We note that, using the non-relativistic Compton cooling in their hydrochemical simulations, Richings \& FaucherGiguère (2018) found that AGN energy-driven outflows can loose the thermalized mechanical energy through efficient cooling and in-situ formation of molecular gas within the outflow, which would imply momentum conservation and energy dissipation similar to what we found for MCG-03-58-007.

Alternatively, the energetics of the molecular outflow might be unrelated to the nuclear X-ray wind. This can be the case if the radiation pressure on the dust of the ISM is the driving mechanism of the large scale outflow (Costa et al. 2018b; Ishibashi et al. 2018). However, this coupling mechanism is most efficient at $L_{\mathrm{AGN}} \gtrsim 10^{47} \mathrm{erg} \mathrm{s}^{-1}$ whereas the $L_{\mathrm{AGN}}$ of MCG-03-58-007 is only $\sim 10^{45} \mathrm{erg} \mathrm{s}^{-1}$. Furthermore, the presence of a nuclear X-ray UFO favours an interpretation in the framework of blast-wave feedback models.

Another possibility, which we cannot test with the current data, is that this putative molecular outflow is driven by a hidden kpcscale radio jet (e.g. see Wagner, Bicknell \& Umemura (2012) for a theoretical framework, and Morganti et al. (2015) for observational evidence). A similar case was recently identified by Husemann et al. (2019) in HE-1353-1917, where the multiphase outflow, including a $\mathrm{CO}$ component, extends by $\sim 1 \mathrm{kpc}$ and has a velocity of $\sim 200 \mathrm{~km} \mathrm{~s}^{-1}$, similar to MCG-03-58-007.

In any case, our results appear to rule out the hypothesis of a momentum-boosted molecular outflow in MCG-03-58-007, despite the presence of a powerful X-ray wind. We note that a similar result, which is at odds with energy conserving models, has been recently obtained for the most luminous local AGN, PDS 456. In this source, Bischetti et al. (2019) detected a large-scale molecular outflow with a momentum rate of the same order of that of the X-ray wind. Furthermore, in IRAS F11119+3257, new ALMA data allowed to revise the estimate of the momentum rate of the molecular outflow, finding that this source may be more consistent with the momentum-conserving feedback scenario, contrary to previous claims (see Veilleux et al. 2017). Finally, Mizumoto et al. (2019) measured a wide range of values for the ratios between the kinetic powers of molecular outflows to the X-ray UFOs, studying a sample of six ULIRGs and quasar-hosts where both an X-ray wind and a molecular outflow have been detected. These findings, together with our new results, point out that the relation (if any) between the disc-wind and the large-scale ISM is more complex than commonly assumed.

\subsubsection{The role of $S F$ feedback}

When comparing the putative molecular outflow energetics with the AGN luminosity and the nuclear wind energetics, we implicitly assumed that all the feedback processes occurring in MCG-03-58007 are due to the AGN activity. In order to test this assumption, we need to verify that the kinetic power output due to star formation activity - stellar radiation and SuperNovae ( $\mathrm{SNe}$ ) explosions - is not sufficient to power the putative molecular outflow. The latter extends up to $R_{\mathrm{OF}} \sim 2 \mathrm{kpc}$ (Table 5). Since we have only a measurement of the total SFR throughout the entire extent of the galaxy, we need to scale down the total SFR to take into account that the putative molecular outflow is much less extended than the molecular disc $\left(R_{\text {gal }} \sim 9 \mathrm{kpc}\right)$. To do so, we use the $L_{\mathrm{CO}}-S F R$ correlation computed for a sample of local SF galaxies (Cicone et al. 2017), which is a direct result of the Schmidt-Kennicutt empirical law (Kennicutt 1998). From the $L_{\mathrm{CO}}$ measured in the nuclear region at $r<(2 \pm 1) \mathrm{kpc}$, we infer $\operatorname{SFR}(r<2 \mathrm{kpc})=10 \pm 5 \mathrm{M}_{\odot} \mathrm{yr}^{-1}$. Using the theoretical predictions of the stellar winds and $\mathrm{SNe}$ output in the ISM (Veilleux, Cecil \& Bland-Hawthorn 2005), which combine stellar evolutionary models, atmospheric models, and empirical spectral data, we find that SF activity can trigger a mass-loss rate of $\dot{M}_{S F}=2.7 \mathrm{M}_{\odot} \mathrm{yr}^{-1}$ within a radius of $2 \mathrm{kpc}$. This is a factor of 20 lower than what we measured for the putative outflow. The expected kinetic power injected into the ISM by SNe is $\dot{E}_{\mathrm{SN}}=7.3 \times 10^{42} \mathrm{erg} \mathrm{s}^{-1}$, which can explain the kinetic energy of the molecular outflow only by assuming a coupling efficiency of 14 per cent. Such efficiency value is at the high end of the range 
allowed by theoretical models. Hence, the energetics of the putative outflow would be hard to reconcile with a purely star formationdriven feedback process.

\subsection{Case II: a kpc-scale rotating structure}

An alternative interpretation for the $\mathrm{CO}(1-0)$ residuals shown in Fig. 10 would be the presence of a compact structure that is rotating possibly with a different geometry and kinematics with respect to the rotation of the larger molecular disc modelled with 3D BAROLO. The compact $\mathrm{CO}(1-0)$ emission shown in Fig. 10 resembles the shape of a doughnut, which may trace a ring with a radius of $R \simeq 1.7 \mathrm{kpc}$, rotating with LOS velocities of about $v_{\text {los }} \simeq 170 \mathrm{~km} \mathrm{~s}^{-1}$ and having a molecular mass of $M_{H 2}^{\text {ring }} \simeq 8 \frac{\alpha_{C O}}{3.1} \times 10^{8} \mathrm{M}_{\odot}$. However, it is difficult to infer the inclination and position angle of such putative ring, for the following reasons:

(i)The limited spatial resolution of the ALMA data does not allow us to constrain the geometry of this structure;

(ii)The CO residual emission in Fig. 10, especially the components that are aligned along the major axis of the main molecular disc fitted by 3D BAROLO (NW-SE direction), might still include a contamination from such rotation;

(iii)The kinematics of the CO residuals (Fig. 11) is likely more complex than a pure rotation pattern and we can provide only a zeroth order description.

Compact rotating structures have been detected at smaller scales $(\sim 10-100 \mathrm{pc})$ in a few Seyfert 2 galaxies and are often denominated circumnuclear discs (CNDs). A closed asymmetrical elliptical ring has been detected in NGC 1068 using maps of $\mathrm{CO}(3-2)$ line emission (García-Burillo et al. ) and $\mathrm{C}_{2} \mathrm{H}$ (García-Burillo et al. 2017); this CND has a radius of $r \sim 200 \mathrm{pc}$ and is off-centred relative to the location of the AGN. Izumi et al. (2018) found a $74 \times 34$ pc CND with a molecular mass of $M_{H 2} \sim 3 \times 10^{6} \mathrm{M}_{\odot}$ in Circinus, the nearest type 2 Seyfert galaxy. Combes et al. (2019) have recently detected six CNDs in a sample of seven local galaxies, with radii ranging from 6 to $27 \mathrm{pc}$ and masses from $0.7 \times 10^{7}$ to $3.9 \times 10^{7} \mathrm{M}_{\odot}$. All six structures show an orientation along the line of sight that is unaligned to that of the host galaxy and, except in one case, they are off-centred with respect to the AGN (Combes et al. 2019). We note however that the structure detected in MCG-03-58-007 is a factor of $\sim 10-100$ larger in size and $\mathrm{H}_{2}$ mass, compared to typical CNDs detected so far. Based on the comparison with previously detected CNDs, we favour the outflow interpretation for the compact residual CO emission in MCG-03-58007, but a rotating structure cannot be ruled out based on the current ALMA data.

\section{SUMMARY AND CONCLUSIONS}

In this work we reported the ALMA CO(1-0) observations of MCG03-58-007 $\left(z_{\mathrm{CO}}=0.03236 \pm 0.00002\right.$, this work $)$, a Seyfert 2 galaxy that we selected as a suitable candidate for investigating AGN feedback mechanisms because of the presence of an extremely powerful X-ray disc-wind (Braito et al. 2018; Matzeu et al. 2019). The target galaxy has a moderate SFR of $20.1 \pm 0.9 \mathrm{M}_{\odot} \mathrm{yr}^{-1}$, which makes it one of the few sources in this more typical SF regime where the blast-wave AGN feedback scenario has been tested, and so where the possible contamination from SF feedback processes is expected to be minimized.

The ALMA data that we presented probe the distribution and kinematics of the molecular ISM in the whole galaxy up to scales of $\sim 20 \mathrm{kpc}$. At the sensitivity of our data, we do not detect any signature of very high-velocity molecular outflows $(v$ $\left.>250 \mathrm{~km} \mathrm{~s}^{-1}\right)$. The $\mathrm{CO}(1-0)$ emission is dominated by rotation in a molecular disc, which we modelled using the software 3D BAROLO, measuring a molecular mass of $M^{\text {disc }}=(7 \pm 5) \times 10^{9} \mathrm{M}_{\odot}$, corresponding to 78 percent of the total molecular gas mass in this galaxy.

However, the model does not properly describe the complex $\mathrm{CO}(1-0)$ kinematics in the central region of MCG-03-58-007. The data-to-model residuals show a compact structure (within $2 \mathrm{kpc}$ from the galaxy centre) with a $20 \sigma$ significance, whose emission shows two symmetric peaks at $v \sim-170 \mathrm{~km} \mathrm{~s}^{-1}$ and $v \sim$ $+170 \mathrm{~km} \mathrm{~s}^{-1}$. This could be the signature of a low velocity molecular outflow. Under this hypothesis, we applied a simple biconical model and estimated the LOS velocity $\left(v=170 \pm 4 \mathrm{~km} \mathrm{~s}^{-1}\right)$, mass-loss rate $\left(\dot{M}_{\text {out }} \sim 54 \mathrm{M}_{\odot} \mathrm{yr}^{-1}\right)$, momentum rate $\left(\dot{P}_{\text {out }} \sim 8 \times\right.$ $\left.10^{34} \mathrm{~g} \mathrm{~cm} \mathrm{~s}^{-2}\right)$, and kinetic power $\left(\dot{E}_{\text {out }} \sim 1.0 \times 10^{42} \mathrm{erg} \mathrm{s}^{-1}\right)$ of such putative molecular outflow. By comparing these values with the energetics of the X-ray disc-wind, we conclude that the energy coupling between the nuclear wind and the large-scale ISM is not as efficient as it is predicted for the more extreme outflows detected in some ULIRGs (Cicone et al. 2014; Fiore et al. 2017; Fluetsch et al. 2019). In the theoretical framework of the blastwave AGN feedback scenario (King 2010; Faucher-Giguère \& Quataert 2012), our results would be more consistent with a momentum-driven outflow rather than an energy-driven outflow. On the other hand, models invoking the effect of AGN radiation pressure on dusty clouds may provide an alternative explanation for the modest energetics of the putative molecular outflow (Costa et al. 2018b; Ishibashi et al. 2018), although they would not explain the simultaneous presence of a powerful X-ray UFO in this source. With an estimated $\operatorname{SFR}(r<2 \mathrm{kpc})=10 \pm 5 \mathrm{M}_{\odot} \mathrm{yr}^{-1}$, we cannot rule out a significant contribution of SF activity to the driving mechanism. However, in the hypothesis of a purely SF-driven outflow, the required coupling efficiency would be very high, i.e. 7 per cent. Moreover, star formation feedback cannot be responsible for the nuclear X-ray UFO.

The low-velocity molecular outflow hypothesis is not the only interpretation for the compact $\mathrm{CO}$ residual emission detected in MCG-03-58-007. Indeed, the current ALMA data do not allow us to rule out that such structure traces a rotating ring or disc whose kinematics is decoupled from the main molecular disc (and so not accounted for by the 3D BAROLO modelling). However, the size and mass of this putative rotating structure in MCG-0358-007 would exceed by a factor of 10-100 the typical values measured for CNDs, which have been detected in other Seyfert galaxies (García-Burillo et al. ; Izumi et al. 2018; Combes et al. 2019). Moreover, in this CND hypothesis, MCG-03-58-007 would show neither a high-velocity nor a low-velocity molecular outflow, which would be even more bizarre given the presence of a powerful $\mathrm{X}$-ray wind and of a kpc-scale ionized outflow (Braito et al. 2018).

Our results, together with other recent works (Veilleux et al. 2017; Cicone et al. 2018; Bischetti et al. 2019; Fluetsch et al. 2019) show that the interpretation of molecular outflows in the framework of AGN feedback models is becoming more and more complicated as we increase sample sizes and study different types of galaxies. However, the targets studied so far do not constitute a complete and unbiased sample, which is necessary to investigate whether massive outflows are regulated by a universal mechanism and, if so, to construct a physical model that describes such mechanism. 


\section{ACKNOWLEDGEMENTS}

This project has received funding from theEuropean Union's Horizon 2020 research and innovation programme under the Marie Sklodowska Curie grant agreement No. 664931. This paper makes use of the following ALMA data: ADS/JAO.ALMA\#2016.1.00694.S. ALMA is a partnership of ESO (representing its member states), NSF (USA), and NINS (Japan), together with NRC (Canada), MOST and ASIAA (Taiwan), and KASI (Republic of Korea), in cooperation with the Republic of Chile. The Joint ALMA Observatory is operated by ESO, AUI/NRAO, and NAOJ. We thank Paola Andreani, Roberto Maiolino, and Fabio Castagna for the fruitful discussions. Gabriele Matzeu is supported by the European Space Agency (ESA) Research Fellowships. We thank the referee for providing insightful comments that helped improve the paper.

\section{REFERENCES}

Aalto S., Garcia-Burillo S., Muller S., Winters J. M., van der Werf P., Henkel C., Costagliola F., Neri R., 2012, A\&A, 537, A44

Alatalo K. et al., 2011, ApJ, 735, 88

Bieri R., Dubois Y., Rosdahl J., Wagner A., Silk J., Mamon G. A., 2017, MNRAS, 464, 1854

Bischetti M. et al., 2019, A\&A, 628, A118

Braito V. et al., 2018, MNRAS, 479, 3592

Cicone C., Feruglio C., Maiolino R., Fiore F., Piconcelli E., Menci N., Aussel H., Sturm E., 2012, A\&A, 543, A99

Cicone C. et al., 2014, A\&A, 562, A21

Cicone C. et al., 2017, A\&A, 604, A53

Cicone C. et al., 2018, ApJ, 863, 143

Combes F. et al., 2013, A\&A, 558, A124

Combes F. et al., 2019, A\&A, 623, A79

Costa T., Rosdahl J., Sijacki D., Haehnelt M. G., 2018a, MNRAS, 473, 4197

Costa T., Rosdahl J., Sijacki D., Haehnelt M. G., 2018b, MNRAS, 479, 2079

Di Matteo T., Springel V., Hernquist L., 2005, Nature, 433, 604

Di Teodoro E. M., 2015, PhD thesis, Univ. Bologna

Di Teodoro E. M., Fraternali F., 2015, MNRAS, 451, 3021

Faucher-Giguère C.-A., Quataert E., 2012, MNRAS, 425, 605

Feruglio C., Maiolino R., Piconcelli E., Menci N., Aussel H., Lamastra A., Fiore F., 2010, A\&A, 518, L155

Feruglio C. et al., 2015, A\&A, 583, A99

Fiore F. et al., 2017, A\&A, 601, A143

Fischer J. et al., 2010, A\&A, 518, L41

Fluetsch A. et al., 2019, MNRAS, 483, 4586

Fontanot F., De Lucia G., Monaco P., Somerville R. S., Santini P., 2009, MNRAS, 397, 1776

Fraternali F., van Moorsel G., Sancisi R., Oosterloo T., 2002, AJ, 123, 3124

García-Burillo S. et al., 2014, A\&A, 567, A125

García-Burillo S. et al., 2015, A\&A, 580, A35

García-Burillo S. et al., 2017, A\&A, 608, A56
Gofford J., Reeves J. N., McLaughlin D. E., Braito V., Turner T. J., Tombesi F., Cappi M., 2015, MNRAS, 451, 4169

González-Alfonso E. et al., 2017, ApJ, 836, 11

Gruppioni C. et al., 2016, MNRAS, 458, 4297

Harrison C. M., Costa T., Tadhunter C. N., Flütsch A., Kakkad D., Perna M., Vietri G., 2018, Nat. Astron., 2, 198

Hopkins P. F., Elvis M., 2010, MNRAS, 401, 7

Husemann B. et al., 2019, A\&A, 627, A53

Ishibashi W., Fabian A. C., 2015, MNRAS, 451, 93

Ishibashi W., Fabian A. C., Maiolino R., 2018, MNRAS, 476, 512

Izumi T., Wada K., Fukushige R., Hamamura S., Kohno K., 2018, ApJ, 867, 48

Jones D. H. et al., 2009, MNRAS, 399, 683

Kennicutt Jr. R. C., 1998, ApJ, 498, 541

King A. R., 2010, MNRAS, 402, 1516

Kormendy J., Ho L. C., 2013, ARA\&A, 51, 511

Leroy A. K. et al., 2015, ApJ, 814, 83

Matzeu G. A. et al., 2019, MNRAS, 483, 2836

Mizumoto M., Izumi T., Kohno K., 2019, ApJ, 871, 156

Morganti R., Oosterloo T., Oonk J. B. R., Frieswijk W., Tadhunter C., 2015, A\&A, 580, A1

Nakai N., Hayashi M., Handa T., Sofue Y., Hasegawa T., Sasaki M., 1987, PASJ, 39, 685

Oosterloo T., Raymond Oonk J. B., Morganti R., Combes F., Dasyra K., Salomé P., Vlahakis N., Tadhunter C., 2017, A\&A, 608, A38

Papastergis E., Cattaneo A., Huang S., Giovanelli R., Haynes M. P., 2012, ApJ, 759, 138

Pereira-Santaella M. et al., 2018, A\&A, 616, A171

Reeves J. N., O'Brien P. T., Ward M. J., 2003, ApJ, 593, L65

Richings A. J., Faucher-Giguère C.-A., 2018, MNRAS, 474, 3673

Sandstrom K. M. et al., 2013, ApJ, 777, 5

Schawinski K. et al., 2014, MNRAS, 440, 889

Sijacki D., Springel V., Di Matteo T., Hernquist L., 2007, MNRAS, 380, 877

Silk J., Rees M. J., 1998, A\&A, 331, L1

Solomon P. M., Vanden Bout P. A., 2005, ARA\&A, 43, 677

Spoon H. W. W. et al., 2013, ApJ, 775, 127

Strong M., Pedlar A., Aalto S., Beswick R. J., Curran S., Booth R., 2004, MNRAS, 353, 1151

Sturm E. et al., 2011, ApJ, 733, L16

Tombesi F., Sambruna R. M., Reeves J. N., Braito V., Ballo L., Gofford J., Cappi M., Mushotzky R. F., 2010, ApJ, 719, 700

Tombesi F., Meléndez M., Veilleux S., Reeves J. N., González-Alfonso E., Reynolds C. S., 2015, Nature, 519, 436

Veilleux S., Cecil G., Bland-Hawthorn J., 2005, ARA\&A, 43, 769

Veilleux S. et al., 2013, ApJ, 776, 27

Veilleux S., Bolatto A., Tombesi F., Meléndez M., Sturm E., GonzálezAlfonso E., Fischer J., Rupke D. S. N., 2017, ApJ, 843, 18

Wagner A. Y., Bicknell G. V., Umemura M., 2012, ApJ, 757, 136

Zschaechner L. K. et al., 2018, ApJ, 867, 111

This paper has been typeset from a $\mathrm{T}_{\mathrm{E}} \mathrm{X} / \mathrm{L} \mathrm{T} \mathrm{E} \mathrm{X}$ file prepared by the author. 